\section{PSICOLOGIA IBEROAMERICANA}

\section{Psicología lberoamericana}

ISSN: 1405-0943

psicología.iberoamericana@uia.mx

Universidad Iberoamericana, Ciudad de

México

México

Romero Palencia, Angélica; Erari Gil-Bernal, Flor de María Modelo predictivo de personalidad sexual en adultos de la Ciudad de México

Psicología Iberoamericana, vol. 21, núm. 1, enero-junio, 2013, pp. 16-23

Universidad Iberoamericana, Ciudad de México

Distrito Federal, México

Disponible en: http://www.redalyc.org/articulo.oa?id=133929862003

Cómo citar el artículo

- Número completo

- Más información del artículo

- Página de la revista en redalyc.org

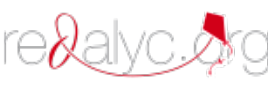

Sistema de Información Científica

Red de Revistas Científicas de América Latina, el Caribe, España y Portugal

Proyecto académico sin fines de lucro, desarrollado bajo la iniciativa de acceso abierto 


\title{
Modelo predictivo de personalidad sexual en adultos de la Ciudad de México
}

\author{
A Predictive Model of Sexual Personality in Adults of Mexico City
}

\author{
Angélica Romero Palencia* \\ Flor de María Erari Gil-Bernal \\ Universidad Autónoma del Estado de Hidalgo
}

\section{RESUMEN}

Diversas investigaciones han demostrado reiteradamente que la personalidad influye en el repertorio conductual que exhiben las personas, y la conducta sexual no es la excepción. De aquí el interés por conocer la influencia de algunos rasgos de personalidad sexual y su relación con la infidelidad, la orientación socio-sexual y la satisfacción en la relación de pareja sobre la personalidad sexual de hombres mexicanos. La muestra estuvo comprendida por 334 hombres adultos voluntarios, de entre 18 y 64 años de edad, los cuales tenían una relación de pareja al momento en que se recolectó la información. Los resultados arrojaron que los predictores más importantes de la personalidad sexual en hombres mexicanos fueron los relacionados con la conducta sexual. Los hallazgos se discuten en términos de las características de la muestra y de los hallazgos en otros estudios.

Palabras clave: personalidad sexual, hombres, mexicanos.

\section{ABSTRACT}

Research has repeatedly shown that personality influences the behavioral repertoire exhibited by persons, and sexual behavior is no exception. Therefore there is interest to know the influence of certain sexual personality traits and their relation with infidelity, socio-sexual orientation and couple relationship satisfaction on the sexual personality of Mexican men. The sample included 334 male adult volunteers, between 18 and 64 years of age, who had a relationship at the time of data collection. Results revealed that the most important predictor of sexual personality in Mexican men were sexual behavior. The findings are discussed in terms of the characteristics of the sample and the findings in other studies.

Keywords: sexual personality, Mexican, men.

\footnotetext{
* Recibido: 6 de noviembre, 2012 - Aceptado: 13 de febrero, 2013

Correspondencia: Angélica Romero Palencia, Instituto de Ciencias de la Salud, Área Académica de Psicología, Universidad Autónoma del Estado de Hidalgo, C. P. 42000, colonia Centro, Pachuca Hidalgo, México.

e-mail: aacrom@gmail.com
} 


\section{INTRODUCCIÓN}

Para Eysenck (1976) la personalidad se define como: "una organización más o menos estable y duradera del carácter, temperamento e intelecto y físico de una persona, que determina su adaptación única al ambiente. El carácter denota el sistema de una persona más o menos estable y duradero del comportamiento conativo (voluntad); el temperamento, el sistema más o menos estable y duradero del sistema del comportamiento afectivo (emoción); el intelecto, el sistema más o menos estable y duradero del comportamiento cognitivo (inteligencia); y el físico, el sistema más o menos estable y duradero de la configuración corporal y de la dotación neuroendocrina" (p. 2) postulados con los que también coinciden Díaz-Loving, Rivera, Rocha y Sánchez Aragón (2002) y Sato (2005).

En sus múltiples aportaciones, Cattel (1950) identifica originalmente 16 rasgos primarios en el ser humano. Extensiones de este trabajo llevaron a Costa y McRae (1992) a postular la existencia de cinco grandes rasgos básicos: extroversión, apertura a la experiencia, responsabilidad, amabilidad, inestabilidad emocional y neuroticismo; mientras que Eysenck (1971) postula tres tipos de personalidad: extroversión, neuroticismo y psicoticismo que posteriormente también fueron retomados por Rammstedt (2013).

La investigación que se ha realizado acerca de las diferencias individuales en sexualidad arroja evidencia de que estas últimas podrían no ser necesariamente independientes de los factores tradicionales de la personalidad estudiados hasta la fecha. Eysenck (1971) y Rowatt (2003) encontraron que las actitudes hacia la sexualidad correlacionaron modestamente con el nivel de extroversión, neuroticismo y psicoticismo. Por ejemplo, las personas extrovertidas tendían a tener actitudes más favorables que las introvertidas con respecto a tener varias parejas sexuales y practicar distintas posiciones sexuales. Aquellos participantes que resultaron altos en neuroticismo tendieron a estar menos satisfechos con sus vidas sexuales que aquéllos que puntuaban bajo en esa misma dimensión (Schmitt, 2004).

Por su parte, Wright y Reise (1997) encontraron que las personas que tenían alto puntaje en extroversión, bajo en agradabilidad y bajo en neuroticismo, tendieron a estar orientadas hacia la elección de parejas de corta duración.
De acuerdo con la Teoría evolutiva de la personalidad, la variación en el ejercicio de la sexualidad y de las tendencias de selección de pareja puede ser una diferencia individual (Buss, 1991; 2000; 2005). Por ejemplo, las diferencias con respecto al atractivo sexual están vinculadas con el éxito para atraer a una pareja deseable (Gangestad, Haselton \& Buss, 2006; Gangestad \& Simpson, 2000; Kurzban \& Aktipis, 2007; Simpson, Gangestad, Christensen \& Leck, 1999); las diferencias con respecto a la exclusividad en una relación afectan el grado de certeza de un padre acerca de la paternidad de su hijo (Symons, 1979); las diferencias en el involucramiento emocional indican la profundidad de una relación y tienen un impacto en la armonía o en la disolución de una relación (Buss, 1994; 2005).

Se ha encontrado que algunos rasgos de personalidad están asociados con la infidelidad (Schmitt, 2004). Tanto Buss y Shackelford y Buss (1997), como Romero, Cruz y Díaz-Loving (2008) descubrieron vínculos fuertes y consistentes entre la personalidad y la propensión a la infidelidad. Buunk y Van Driel (1989) y Simpson et al. (1999) encontraron que las aventuras han sido atribuidas al narcisismo, a la necesidad de reafirmar un ego masculino frágil y de elevar la autoestima y, en general, al mal funcionamiento psicológico.

Miller et al. (2000) estudiaron las dimensiones de Eysenck y las de los "Cinco grandes" de la personalidad y hallaron que la baja conciencia y los niveles altos de narcicismo predicen el deseo de tener múltiples parejas sexuales, sobre todo en las mujeres, mientras que la apertura es un predictor de la susceptibilidad a la infidelidad en los hombres, y el psicoticismo se relaciona positiva y significativamente con la infidelidad, tanto en hombres como en mujeres.

En un estudio previo, Romero (2007) detectó que los factores de personalidad sexual se agrupaban en tres grandes categorías: la personalidad sexual conquistadora, que se caracteriza por una persona involucrada, amorosa, conquistadora y romántica; la personalidad sexual abierta, cuyas características básicas son una orientación socio-sexual abierta con amplio número de parejas sexuales, infiel y masculina, y la personalidad sexual restrictiva, que es tradicional, moralista, virginal y restringida. Por lo anterior, el objetivo de la presente investigación consistió en detectar si factores tales como la infidelidad, la orientación socio-sexual y 
la satisfacción con la relación son predictores válidos y confiables de la personalidad sexual conquistadora, abierta o restrictiva de hombres mexicanos.

\section{MÉTODO}

\section{Participantes}

Para la realización de esta investigación se contó con la colaboración de 334 varones adultos voluntarios a través de un muestreo no probabilístico. La edad osciló entre los 18 y los 64 años, con una media de 30 años $(\mathrm{DE}=9.66)$. Todos los participantes estaban involucrados en una relación de pareja heterosexual. El $53 \%$ informó no haber tenido una vivencia previa de infidelidad, mientras que el resto (47\%) dijo que sí la había tenido.

\section{Instrumentos}

- Inventario de Orientación Sociosexual (García \& Díaz-Loving, 2007)

Está integrado por siete reactivos, de los cuales tres son preguntas abiertas y cuatro son afirmaciones tipo Likert de nueve puntos y un alfa de Cronbach $=.96$ que evalúa dos dimensiones: Orientación Sociosexual Actitudinal y Orientación Sociosexual Conductual.

- Escala de Motivación Sexual (García \& DíazLoving, 2007)

Está integrada por 67 reactivos tipo Likert de cinco puntos, distribuidos en dos subescalas: la Subescala de Inhibición Sexual formada por 37 reactivos, que cuenta con tres factores: vinculación deficiente, falta de deseo y obstáculos, y la Subescala de Motivación Sexual, compuesta por 30 reactivos que se agrupan en cinco factores: expresión afectiva, atracción interpersonal, placer físico, deseo sexual y facilitadores. Con un alfa de Cronbach $=.948$.

- Inventario de Conducta Sexual (García \& DíazLoving, 2007)

Está compuesto de 73 reactivos tipo Likert con siete opciones de respuesta y un a de Cronbach $=.96$. Mide cinco dimensiones de la conducta sexual, que son: contacto sexual, seducción, autoerotismo, contacto físico, variantes sexuales.

- Escala de Personalidad Sexual (Díaz-Loving, Rivera, Rocha, Sánchez \& Schmitt, 2002) Está compuesta por 89 reactivos tipo Likert con nueve opciones de respuesta (que va desde "me describe extremadamente exacto", hasta "me describe extremadamente inexacto"). Los coeficientes de confiabilidad de todos los factores oscilaron entre .51 y .94. y mide 10 dimensiones de personalidad sexual, que son: atractivo involucrado, perverso, atractivo sexual, conquistador, liberal, infiel, masculinidad, restricción sexual, amoroso, y diversidad sexual.

- Versión Corta de la Escala de Satisfacción Marital (Cortés, Reyes, Díaz-Loving, Rivera \& Monjaraz, 1994)

Está compuesta por 48 reactivos tipo Likert con cinco opciones de respuesta y un a de Cronbach $=.91$. Evalúa seis dimensiones de la satisfacción marital que son: interacción físico-sexual, organización, familia, diversión e hijos.

- Subescala de Conducta Infiel del Inventario Multidimensional de Infidelidad (Romero, Rivera \& Díaz-Loving, 2007)

Tiene 35 reactivos tipo Likert con cinco opciones de respuesta y un alfa de Cronbach $=.98$. Mide cuatro dimensiones de infidelidad, que son: infidelidad sexual, infidelidad emocional, deseo de infidelidad sexual y deseo de infidelidad emocional.

\section{Procedimiento}

Se pidió a los participantes que respondieran a las escalas de la manera más clara y sincera posible, indicando que el cuestionario formaba parte de una investigación cuyo objetivo era conocer características de las relaciones de pareja. Asimismo, se destacó el anonimato de las respuestas y se les indicó que éstas no serían catalogadas como buenas o malas, correctas o incorrectas, lo anterior con la finalidad de garantizar, en la medida de lo posible, la honestidad de los participantes. 


\section{RESULTADOS}

Una vez aplicados los instrumentos a los participantes y dado el extenso número de factores de las escalas se realizó un análisis factorial de segundo orden, incluyendo la totalidad de factores. El análisis factorial de componentes principales con rotación ortogonal arrojó 16 factores con autovalores mayores a uno que explicaron el $75.8 \%$ de la varianza. Los factores obtenidos fueron: motivación sexual ( $\alpha=.94)$, satisfacción con la relación primaria $(\alpha=.90)$, personalidad conquistadora involucrada $(\alpha=.90)$, motivos asociados a la personalidad $(\alpha=.90)$, infidelidad emocional $(\alpha=.77)$, desmotivación sexual $(\alpha=.89)$, personalidad sexual abierta $(\alpha=.73)$, conducta sexual $(\alpha=.87)$, infidelidad sexual $(\alpha=.78)$, variantes sexuales $(\alpha=.78)$ e insatisfacción $(\alpha=.74)$ (Ver tabla 1).

\section{Tabla 1. Factores de segundo orden obtenidos}

\begin{tabular}{|c|c|c|}
\hline Factor & Alfa & Indicadores \\
\hline Motivación sexual & .936 & $\begin{array}{l}\text { Atracción, ambiente propicio, expresión afectiva, } \\
\text { placer físico. }\end{array}$ \\
\hline Satisfacción con la relación primaria & .897 & $\begin{array}{l}\text { Diversión, área físico-sexual, organización } \\
\text { conjunta, interacción. }\end{array}$ \\
\hline Infidelidad emocional & .892 & Enamoramiento, infidelidad emocional. \\
\hline Motivos asociados a la personalidad & .892 & $\begin{array}{l}\text { Impulsividad, inestabilidad social y emocional, } \\
\text { ideología tradicional. }\end{array}$ \\
\hline $\begin{array}{l}\text { Conceptuación negativa de la } \\
\text { infidelidad }\end{array}$ & .772 & Trasgresión a la relación, traición, dolor, engaño. \\
\hline Desmotivación sexual & .889 & $\begin{array}{l}\text { Falta de afecto, vinculación deficiente, falta de } \\
\text { interés, obstáculos. }\end{array}$ \\
\hline Infidelidad sexual & .730 & $\begin{array}{l}\text { Sexo sin amor, sexo de una noche, múltiples } \\
\text { parejas sexuales. }\end{array}$ \\
\hline Conducta sexual & .874 & Contacto físico, contacto sexual, seducción. \\
\hline Conceptuación positiva de la infidelidad & .729 & Placer, pasión, variedad, satisfacción. \\
\hline Variantes sexuales & .775 & Pornografía, masturbación, juguetes sexuales. \\
\hline Insatisfacción & .742 & Insatisfacción, aburrimiento, apatía. \\
\hline Deseo de infidelidad emocional & .741 & Deseo de enamorarse de alguien más. \\
\hline Deseo de infidelidad sexual & .740 & Deseo de tener contacto sexual con alguien más. \\
\hline
\end{tabular}


Posteriormente se llevaron a cabo análisis de regresión jerárquica múltiple paso por paso para detectar cuáles factores serían predictores confiables de la personalidad sexual en hombres.

\section{Personalidad sexual conquistadora}

El análisis de regresión constó de dos pasos en los que se detectó que los predictores más importantes fueron la Conducta sexual y la Infidelidad emocional. Aquellos hombres que presentaron una alta frecuencia de conductas de tipo sexual, tales como el tocamiento físico íntimo, incluyendo el preludio sexual, el sexo coital y oral y elementos de fantasía y comunicación, así como el hecho de enamorarse constantemente de otras personas además de su pareja, indicaron ser determinantes para que desarrollaran y mantuvieran una persoalidad sexual conquistadora.

\section{Personalidad sexual abierta}

En el caso de la personalidad sexual abierta, el análisis de regresión múltiple constó de siete pasos. Se detectó que los predictores más importantes fueron aquellos que involucraban la relación de pareja. Los hombres de la muestra que presentaban una personalidad sexual abierta contaban con menor satisfacción con la relación, desconfianza hacia su pareja, deseo de infidelidad y una personalidad sexual descrita como inestable (ver tabla 3).

\section{Personalidad sexual restrictiva}

Finalmente, para la personalidad sexual restrictiva el análisis de regresión múltiple constó de cinco pasos. Se detectó que los predictores más importantes fueron aquellos que involucraban la sexualidad. Los hombres de la muestra con una concepción negativa de la in-

Tabla 2. Predictores confiables para la Personalidad sexual conquistadora

\begin{tabular}{l|c|c|c|c|c|c|c}
\hline Modelo & B & Sig. & $\begin{array}{c}\text { Cambio } \\
\text { de la F }\end{array}$ & $\begin{array}{c}\text { Sig. del cambio } \\
\text { de la F }\end{array}$ & R Múltiple & $\mathbf{R}^{2}$ & $\begin{array}{c}\text { Error } \\
\text { estándar }\end{array}$ \\
\hline Conducta sexual & .561 & .000 & 12.387 & .001 & .574 & .320 & .994 \\
\hline Infidelidad emocional & -.350 & .001 & & & & \\
\hline
\end{tabular}

Tabla 3. Predictores confiables para la Personalidad sexual abierta

\begin{tabular}{|c|c|c|c|c|c|c|c|c|}
\hline Modelo & & B & Sig. & $\begin{array}{c}\text { Cambio } \\
\text { en } F\end{array}$ & $\begin{array}{c}\text { Sig. del } \\
\text { cambio de la F }\end{array}$ & $\begin{array}{c}\mathbf{R} \\
\text { Múltiple }\end{array}$ & $\mathbf{R}^{2}$ & $\begin{array}{c}\text { Error } \\
\text { estándar }\end{array}$ \\
\hline \multirow[t]{7}{*}{7} & $\begin{array}{l}\text { Deseo de infidelidad } \\
\text { emocional }\end{array}$ & .311 & .006 & 6.119 & .015 & .758 & .555 & .779 \\
\hline & $\begin{array}{l}\text { Satisfacción con } \\
\text { la relación }\end{array}$ & -.229 & .000 & & & & & \\
\hline & Desconfianza & .244 & .000 & & & & & \\
\hline & $\begin{array}{l}\text { Deseo de infidelidad } \\
\text { sexual }\end{array}$ & .326 & .004 & & & & & \\
\hline & Personalidad inestable & .247 & .001 & & & & & \\
\hline & $\begin{array}{l}\text { Motivos asociados } \\
\text { a la sexualidad }\end{array}$ & .166 & .005 & & & & & \\
\hline & $\begin{array}{l}\text { Insatisfacción con } \\
\text { la relación }\end{array}$ & .160 & .015 & & & & & \\
\hline
\end{tabular}


fidelidad, con baja motivación sexual, alta desmotivación y baja conducta sexual y una personalidad descrita como inmadura e inestable, tenderán con mayor probabilidad a presentar una personalidad sexual restrictiva (ver tabla 4 ).

\section{DISCUSIÓN}

A partir de los resultados encontrados en la presente investigación podemos inferir que el desarrollo de una personalidad sexual estará íntimamente relacionado con el objetivo de la conducta sexual (Buss, 2005).

Como se puede observar, la personalidad sexual conquistadora tuvo como principales predictores la conducta sexual y el deseo de infidelidad emocional. Esto indica que los hombres de esta muestra se mostrarán conquistadores cuando tengan una alta frecuencia de conducta sexual y un deseo de enamorarse de una nueva pareja, además de la que ya tienen.

En el caso de la personalidad sexual abierta influirá principalmente el grado de insatisfacción que tengan con su relación actual, la percepción de inestabilidad, la concepción positiva hacia tener varias parejas sexuales a la vez y el grado de celos y desconfianza que tengan hacia su pareja actual.

Finalmente, en el caso de la personalidad sexual restrictiva se puede observar que también influye la autopercepción de inestabilidad y la baja motivación sexual, así como un concepto negativo hacia tener varias relaciones simultáneas.
Es de resaltar el hecho de que las características típicas de personalidad asociadas con la impulsividad, la inestabilidad social y emocional y la ideología tradicional hayan tomado relevancia en el estudio, primero conformándose en un factor de segundo orden y después al resultar ser predictores importantes, tanto con el desarrollo de una personalidad sexual abierta como restrictiva; no así con la personalidad sexual conquistadora. Esto corrobora, como lo menciona la literatura previa, la influencia de características neuróticas en el desarrollo de la personalidad sexual (Eysenck, 1976; Miller, Lynam, Zimmerman, Logan, Leukefeld \& Clayton, 2000; Wright \& Reise, 1997).

El hecho de autopercibirse como una persona inestable e impulsiva puede orientar a un hombre a desarrollar una personalidad sexual abierta cuando se encuentre insatisfecho en su relación actual, sienta celos y desconfianza hacia su pareja y tenga un fuerte deseo de tener otras parejas emocionales o sexuales. Por el contrario, las mismas características de impulsividad e inestabilidad podrían orientar a una persona a desarrollar una personalidad sexual restrictiva cuando tenga baja motivación sexual, poca frecuencia de conducta sexual y un concepto negativo de la infidelidad.

Es así como encontramos que el desarrollo de la personalidad sexual se encuentra íntimamente ligado con el objetivo que se persigue y la adaptación al entorno (Buss, 2005).

Tabla 4. Predictores confiables para la Personalidad sexual restrictiva

\begin{tabular}{c|l|c|c|c|c|c|c|c}
\hline Modelo & & B & Sig. & $\begin{array}{c}\text { Cambio } \\
\text { en F }\end{array}$ & $\begin{array}{c}\text { Sig. del } \\
\text { cambio de la F }\end{array}$ & $\begin{array}{c}\mathbf{R} \\
\text { Múltiple }\end{array}$ & $\mathbf{R}^{2}$ & $\begin{array}{c}\text { Error } \\
\text { estándar }\end{array}$ \\
\hline 5 & Personalidad inestable & .247 & .001 & 4.279 & .040 & .535 & .286 & 1.23 \\
\hline & $\begin{array}{l}\text { Conceptuación positiva } \\
\text { de la infidelidad }\end{array}$ & -.211 & .006 & & & & & \\
\hline & Motivación sexual & -.396 & .000 & & & & & \\
\hline & Desmotivación sexual & .303 & .000 & & & & & \\
\hline
\end{tabular}


Un hombre típico de la muestra se mostrará conquistador cuando desee experimentar el enamoramiento hasta llegar a la conducta sexual. Se mostrará abierto, atractivo, sensual y seductor cuando busque salir de su relación actual y tener una variedad de experiencias sexuales, tanto en diversidad como en frecuencia. Finalmente, un hombre de esta muestra se mostrará restrictivo, moral y virginal cuando tenga en general una baja conducta sexual tanto en motivación como en frecuencia y un concepto negativo de la infidelidad.

Estos hallazgos indicarían que el desarrollo de la personalidad sexual se encuentra íntimamente ligado con los objetivos sexuales que persiguen los hombres;

\section{REFERENCIAS}

Barash, D. P. \& Lipton, J. E. (2001).The myth of monogamy: Fidelity and infidelity in animals and people. NY: W. H. Freeman and Company.

Buss, D. M. (1991). Evolutionary personality psychology. Annual Review of Psychology, 42, 459-491.

Buss, D. M. (1994). The evolution of desire: Strategies of human mating. NY: Basic Books.

Buss, D. M. (2000). The dangerous passion. NY: The Free Press.

Buss, D. M. (2005). The Handbook of Evolutionary Psychology. NY: John Wiley \& Sons.

Buss, D. M. \& Schmitt, D. P. (1993). Sexual strategies theory: An evolutionary perspective on human mating. Psychological Review, 100, 204-232.

Buunk, B. P. \& Van Driel, B. (1989). Variant lifestyles and relationships. Newbury Park, C.A: Sage.

Buunk, B. P. \& Dijkstra, P. (2000). Extradyadic Relationships and Jealously. En C. Hendrick \& S. Hendrick (Eds.). Close Relationships. A Sourcebook. CA: Sage.

Catell, R. B. (1950). Personality: A systematic, theoretical and factual study. NY: Mc Graw-Hill.

Córtes M. S., Reyes D., D., Díaz-Loving, R., Rivera A., S. \& Monjaraz C., J. (1994). Elaboración y análisis psicométrico del Inventario Multifacético de Satisfacción lo que apoya algunas teorías evolutivas, tales como la de la inversión parental (Buunk \& Dijkstra, 2000; Symons, 1979; Trivers, 1972) y la teoría de las estrategias sexuales (Barash \& Lipton, 2001; Buss \& Schmitt, 1993).

El campo de la personalidad sexual ha sido poco explorado aún. La investigación y los resultados al respecto son escasos. De ahí la relevancia de los resultados empíricos asociados a la personalidad sexual. Es indispensable, por tanto, continuar con el desarrollo de los estudios que vinculen a la personalidad sexual con otras variables y fenómenos de las interacciones sociales, capaces de crear modelos tanto de predicción como de explicación de la personalidad sexual. •

Marital. La Psicología social en México. México: Asociación Mexicana de Psicología Social.

Costa, P. F. Jr. \& McCrae, R. R. (1992). Four Ways five factors are basic. En Personality and Individual Differences 13, 653-665.

Díaz-Loving, R. \& Sánchez Aragón, R. (2002). Psicología del Amor: una visión integral de la relación de pareja. México: Miguel Ángel Porrúa.

Díaz-Loving, R., Rivera Aragón, S., Rocha Sánchez, T. E. \& Sánchez Aragón, R. (2002). Marcado por la conquista: Rasgos de personalidad derivados de la vida sexual. Revista de Psicología Social y Personalidad, 18(2), 77-92.

Eysenck, H. J. (1971). Personality and attitudes to sex: A factorial study. En Personality, 1, 355-376.

Eysenck, H. J. (1976). Sex and personality. London: Open Books.

Gangestad, S. W. \& Simpson, J. A. (2000). The evolution of human mating: Trade-offs and strategic pluralism. Behavioral and Brain Sciences, 23, 573-587.

Gangestad, S.W., Haselton, M. G. \& Buss, D. M. (2006). Evolutionary foundations of cultural variation: evoked culture and mate preferences. Psychological Inquiry, 17(2), 75-95. 
García, G. \& Díaz-Loving, R. (2007) Expresiones afectivas y sexuales en diversos contextos relacionales. En Crisis, expresiones sexuales y celos en las relaciones de pareja. Simposio efectuado en el xxxi Congreso Interamericano de Psicología, Distrito Federal, México.

Kurzban, R. \& Aktipis, C. A. (2007). Modularity and the social mind: Are psychologist too self-ish? Personality and Social Psychology Review, 11(2), 131-149.

Miller, J. D., Lynam, D. R., Zimmerman, R., Logan, T., Leukefeld, C. \& Clayton, R. (2000). Risky sexual behavior and the five factor model of personality. En Meeting of the American Psychological Association. Washington, D. C.

Rammstedt, B. (2013). Correcting Big Five Personality Measurements for Acquiescence: An 18-Country Cross-Cultural Study. European Journal Of Personality, 27(1), 71-81.

Romero, A. (2007). Infidelidad: conceptuación, correlatos $y$ predictores. (Tesis de doctorado inédita). Facultad de Psicología. Universidad Nacional Autónoma de México, México.

Romero, A., Cruz, C. \& Díaz-Loving, R., (2008). Propuesta de un modelo biopsicosociocultural de infidelidad sexual y emocional en hombres y mujeres. Psicología Iberoamericana, 16(2), 14-21.

Romero, A., Rivera S. \& Díaz-Loving, R. (2007). Desarrollo del Inventario Multidimensional de Infidelidad (IMIN). Revista Iberoamericana de Diagnóstico y Evaluación en Psicología, 23(1), 121-148.
Rowatt, W. P. (2003). Associations Between Religious Orientation and Varieties of Sexual Experience. Journal For The Scientific Study of Religion, 42(3), 455-465.

Sato, T. (2005). The Eysenck Personality Questionnaire Brief Version: Factor Structure and Reliability. Journal of Psychology, 139(6), 545-552.

Shackelford, T. K., \& Buss, D. M. (1997). Marital satisfaction in evolutionary psychology perspective. En R. J. Sternberg \& M. Hojjat et al. (Eds.), Satisfaction in close relationships. (pp. 7-25). NY: Guilford Press.

Simpson, J. A., \& Gangestead, S. (1991). Individual differences in sociosexuality: Evidence for convergent and discriminant validity. Journal of Personality and Social Psychology, 59, 1192-1201.

Schmitt, D. P. (2004). The Big Five related to risky sexual behaviour across 10 world regions: differential personality associations of sexual pomiscuity and relationship infidelity. European Journal of Personality, 18(4), 301-319.

Symons, D. (1979). The evolution of human sexuality. NY: Oxford Press.

Trivers, R. (1972). Parental investment and sexual selection. En B. Campbell (Ed). Sexual selection and the descent of man, 1871-1971 (pp. 136-179). Chicago: Adline.

Wright, T. M. \& Reise, S. P. (1997). Personality and unrestricted sexual behavior: correlations of sociosexuality in caucasian and Asian College students. Journal of Research in Personality, 31, 166-192. 